\title{
Effective anchoring of Pt-nanoparticles onto sulfonated polyelectrolyte-wrapped carbon nanotubes for use as a fuel cell electrocatalyst
}

\begin{abstract}
Tsuyohiko Fujigaya $^{1,2}$, ChaeRin $\mathrm{Kim}^{1}$, Kazuya Matsumoto ${ }^{1}$ and Naotoshi Nakashima ${ }^{1,2,3}$
Sulfonated polymers, such as sulfonated polysulfone and sulfonated polyimide, are promising candidates as a future polyelectrolyte for polymer electrolyte fuel cells (PEFCs). In PEFC cell systems, the same polyelectrolyte material is often added to the electrocatalyst layer as the ionic path. We selected partially sulfonated poly(arylene thioethersulfone) (SPTES) and poly[(2,2'-benzidinedisulfonicacid)-alt-(1,4,5,8-naphthalenetetracarboxylic dianhydride)] (BDSA-NTDA) as the electrolytes and wrapped multi-walled carbon nanotubes (MWNTs) with the materials to which platinum nanoparticles (Pt) were attached onto the polyelectrolyte layer to fabricate new electrocatalysts. In these electrocatalysts, the MWNT and Pt serve as the electron path and reaction site, respectively. For both polyelectrolytes, Pt-nanoparticles $<5 \mathrm{~nm}$ in diameter with a narrow size distribution were observed to be homogeneously dispersed on the polymer-wrapped MWNTs. The electrochemical measurements revealed that MWNT/SPTES/Pt and MWNT/BDSA-NTDA/Pt possessed electrochemically active surface areas (ECSA) of 42.3 and $35.6 \mathrm{~m}^{2} / \mathrm{g}$ of $\mathrm{Pt}$, respectively. Such a high ECSA efficiency ensures the use of these composites for the preparation of a future electrocatalyst.

Polymer Journal (2013) 45, 326-330; doi:10.1038/pj.2012.145; published online 1 August 2012
\end{abstract}

Keywords: carbon nanotubes; composite materials; electrocatalysts; fuel cell; platinum; polyelectrolyte

\section{INTRODUCTION}

Carbon nanotubes (CNTs) are attractive materials in many fields of science, including materials science, (bio)technology, and electronics, because of their unique structure, outstanding electronic properties, and mechanical stiffness. Among the wide range of applications for CNTs, the metal nanoparticle/CNTs hybrid is an attractive material, especially for use as fuel cell electrocatalysts owing to the large surface areas, remarkable electrical conductivity and excellent electrochemical durability of the CNTs. ${ }^{1}$ Various metals, such as Pt and Pd, have been immobilized on CNTs and used for fuel cell applications. ${ }^{2,3}$ It is well recognized that pristine CNTs possess insufficient binding sites for anchoring the metal precursors onto their graphitic surfaces, which results in poor dispersion of the particles. ${ }^{4}$ To improve the loading efficiency, surface functionalization of CNTs has been investigated. A common approach to introduce an anchoring unit is the strong oxidation of the CNT to generate carboxyl functional groups. ${ }^{5}$

However, such a covalent functionalization causes structural damage and is often accompanied by reducing the intrinsic properties of CNTs, such as high electrical conductivity and electrochemical durability, which are unfavorable as electrocatalysts for fuel cells. For these reasons, metal anchoring onto the surface of CNTs through non-covalent functionalization is desirable. Several anchoring molecules based on the non-covalent functionalization using 1-aminopyrene, ${ }^{6} \mathrm{DNA}^{7}$ and polypyrrole ${ }^{8}$ have been reported. We have reported that polybenzimidazole (PBI) also serves as an anchoring polymer through the combination of the strong $\pi-\pi$ interactions of PBI on the surfaces of CNTs and the coordination of metal ions with the imidazole group. ${ }^{4,9} \mathrm{PBIs}$ are unique anchoring polymers because they function as a proton conductive polyelectrolyte after acid doping and are considered to be a promising polyelectrolyte for polymer electrolyte fuel cells (PEFCs). As a consequence, the composite of multi-walled carbon nanotubes (MWNTs), PBI and Pt possesses electron conductivity, proton conductivity, and a reaction site, all of which are required for PEFC electrocatalysts. ${ }^{4,9}$ We reported that the use of a polyelectrolyte as the anchoring unit that provides a large exposed surface enables a smooth fuel gas access in PEFCs and provides for an ideal triple-phase interface structure. ${ }^{4,9}$ To date, perfluorosulfonic acid polymers, such as Nafion, have been widely used as a polyelectrolyte for PEFCs, and the development of the Nafion-based electrocatalysts have been extensively studied. However, because of the cost problem and its mechanical weakness, the postNafion is required in PEFCs. Therefore, many extensive studies

${ }^{1}$ Department of Applied Chemistry, Graduate School of Engineering, Kyushu University, 744 Motooka, Nishi-ku, Fukuoka, Japan; ${ }^{2}$ International Institute for Carbon-Neutral Energy Research (WPI-I2CNER), Kyushu University, 744 Motooka, Nishi-ku, Fukuoka, Japan and ${ }^{3} J S T-C R E S T, 5$ Sanbancho, Chiyoda-ku, Tokyo, Japan

Correspondence: Professor T Fujigaya or Professor N Nakashima, Department of Applied Chemistry, Graduate School of Engineering, Kyushu University, 744 Motooka, Nishi-ku, Fukuoka 819-0395, Japan.

E-mail: fujigaya-tcm@mail.cstm.kyushu-u.ac.jp or nakashima-tcm@mail.cstm.kyushu-u.ac.jp

Received 12 April 2012; revised 10 June 2012; accepted 11 June 2012; published online 1 August 2012 
exploring alternative polyelectrolytes, such as PBIs, have been performed. When these polymers are employed as the electrolyte membrane for PEFCs, the same polyelectrolyte is necessary in the electrocatalyst to provide smooth proton conduction between the membrane and electrocatalyst. Hence, the PBI-based electrocatalyst is useful in PEFCs using PBI as a membrane. Indeed, we achieved a high PEFC performance using the composite of MWNT/PBI/Pt as the electrocatalyst, in which the anchored PBI on the MWNTs effectively worked as the proton conducting material in the electrocatalyst. ${ }^{10,11}$

In this study, as shown in Figure 1, we have chosen a sulfonated poly(arylene ethersulfone) and sulfonated polyimide (SPI) (Figure 1) as the polymer anchors because both polymers are also promising alternative polyelectrolytes and have been extensively investigated because of their excellent proton conductivities, mechanical toughness, and low fuel crossover, ${ }^{12}$ and we examined their performance as the electrocatalyst for PEFCs.

\section{EXPERIMENTAL PROCEDURE}

\section{Materials}

$\mathrm{N}, \mathrm{N}$-dimethylacetamide (DMAc), ethylene glycol, and hydrogen hexachloroplatinate hexahydrate $\left(\mathrm{H}_{2} \mathrm{PtCl}_{6} \cdot 6 \mathrm{H}_{2} \mathrm{O}\right)$ were purchased from Wako Pure Chemical (Osaka, Japan) and used as received. Sulfonated poly(arylene ethersulfone) (SPES) and poly(arylene thioethersulfone) (SPTES) were synthesized using previously reported procedures. ${ }^{13-15}$ SPIs were synthesized using the polycondensation reaction of $2,2^{\prime}$-benzidinedisulfonic acid (BDSA) with carboxylic dianhydride according to a reported procedure. ${ }^{16} \mathrm{~A}$ polytetrafluoroethylene (PTFE) filter (pore size, $0.2 \mu \mathrm{m}$; Millipore, Billerica,
MA, USA) was used for filtration. A copper grid with a carbon support (Ouken Shoji, Tokyo, Japan) was used for the TEM (transmission electron microscope) observations. The MWNTs used in this study were kindly supplied by Nikkiso Co. (Tokyo, Japan).

\section{Measurements}

The IR spectra were recorded using a Spectrum 100 FT-IR (Perkin-Elmer, Waltham, MA, USA) spectrometer equipped with an ATR. The TGA (thermogravimetric analysis) curves were measured using an SSC 5200 (SII) operated in conditioned air at a heating rate of $5^{\circ} \mathrm{C} / \mathrm{min}$. The $\mathrm{X}$-ray photoelectron spectroscopy (XPS) measurements were carried out using an AXIS-ULTRA (KRATOS, Shimadzu, Kyoto, Japan) instrument. The binding energy was calibrated using the $\mathrm{C}_{1 \mathrm{~s}}$ peak that appears at $284.5 \mathrm{eV}$. The TEM measurements were conducted using a JEM-2010 (JEOL, Tokyo, Japan) electron microscope operating at $120 \mathrm{kV}$.

\section{Wrapping of MWNTs by polyelectrolytes}

Five milligrams of the MWNTs were added to SPTES (5.0 mg) dissolved in DMAc $(5.0 \mathrm{ml})$ and sonicated for $60 \mathrm{~min}$ using a bath-type sonicator (BRANSON 5510, Sterling Heights, MI, USA). The obtained solution was then centrifuged at $10000 \mathrm{~g}$ to remove any remaining aggregated sediment. The supernatant was filtered and then washed with DMAc to remove any excess SPTES. The obtained solid, denoted MWNT/SPTES, was then dried under a vacuum. The MWNTs wrapped with NTDA-BDSA were prepared using a similar procedure.

\section{Pt-anchoring on MWNT/polyelectrolyte hybrids}

The Pt-nanoparticles were loaded onto MWNT/SPTES by reducing $\mathrm{H}_{2} \mathrm{PtCl}_{6} \cdot 6 \mathrm{H}_{2} \mathrm{O}$ in an aqueous ethylene glycol solution. First, MWNT/SPTES

a Poly(arylene ethersulfone)s

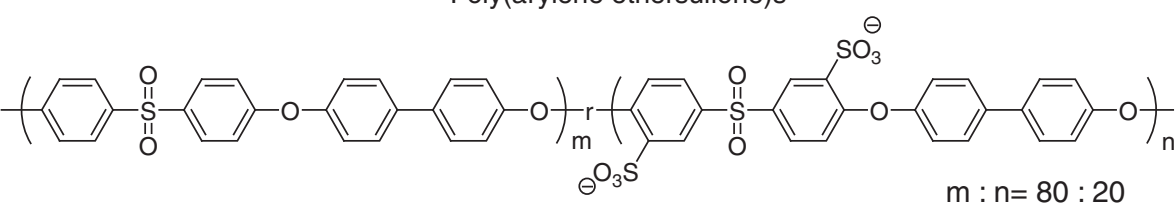

SPES

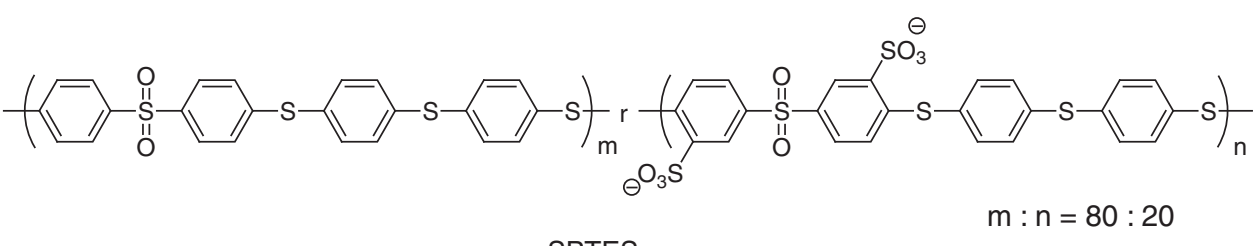

SPTES

b

Polyimides

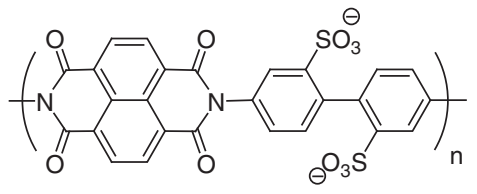

NTDA-BDSA<smiles>Cc1ccc(-c2ccc(N3C(=O)C4CC5C(=O)C6C(=O)N(C)C(=O)C6C5CC4C3=O)cc2[S+](=O)[O-])c(S(=O)(=O)[O-])c1</smiles>

CHDA-BDSA<smiles>Cn1c(=O)c2cc3c(=O)n(-c4ccc(-c5ccccc5[S+](=O)[O-])c([S-](=O)([O-])[O-])c4)c(=O)c3cc2c1=O</smiles>

PDMA-BDSA<smiles>Cc1ccc(-c2ccc(N3C(=O)C4C5C(=O)N(C)C(=O)C5C4C3=O)cc2[S+](=O)[O-])c([S+](=O)[O-])c1</smiles>

CBDA-BDSA

Figure 1 Chemical structures of (a) sulfonated poly(arylene ethersulfone)s and (b) SPIs. 
(10 mg) was added to a $60-$ vol\% aqueous ethylene glycol solution $(20 \mathrm{ml})$ and dispersed by sonication. $\mathrm{H}_{2} \mathrm{PtCl}_{6} \cdot 6 \mathrm{H}_{2} \mathrm{O}(24.0 \mathrm{mg})$ dissolved in the $60-\mathrm{vol} \%$ aqueous ethylene glycol solution $(30 \mathrm{ml})$ was added to the MWNT/SPTES dispersion, and then the mixture was heated at $80^{\circ} \mathrm{C}$ for $6 \mathrm{~h}$. The solid material was collected by filtration and washed with water and then dried under vacuum to obtain MWNT/SPTES/Pt. The Pt-loading on MWNT/NTDABDSA was carried out using a similar procedure to yield MWNT/NTDABDSA/Pt.

\section{Electrochemical measurements}

Electrochemically active surface areas (ECSA) measurements were conducted in a nitrogen-deaerated $0.1 \mathrm{M} \mathrm{HClO}_{4}$ solution. $\mathrm{An} \mathrm{Ag} / \mathrm{AgCl}$ and a Pt wire were used as the reference and counter electrodes, respectively. A 17.6- $\mu$ l aliquot of the DMAc mixture $(1.8 \mathrm{ml})$ and Milli-Q water $(0.2 \mathrm{ml})$ containing $1.0 \mathrm{mg}$ of MWNT/SPTES/Pt or MWNT/NTDA-BDSA/Pt was dropped onto a polished glassy carbon electrode $(6.0 \mathrm{~mm}$ diameter $)$ and then dried. The cyclic voltammogram (CV) was measured at a scan rate of $50 \mathrm{mV} / \mathrm{s}$ at $25^{\circ} \mathrm{C}$ using an electrochemical analyzer 1230A (ALS). The electrode was activated by cycling the potential between -0.13 and $1.0 \mathrm{~V}$ vs $\mathrm{Ag} / \mathrm{AgCl}$ for the ECSA measurements.

\section{RESULTS AND DISCUSSION}

\section{Dispersion of CNTs using polyelectrolytes}

The dispersion abilities of the partially sulfonated poly(arylene ethersulfone)s (SPESs; Figure 1a) and SPIs (Figure 1b) for the dispersion of the MWNTs were investigated. Compared with Nafion, both polymers are known to possess a higher proton conductivity and greater mechanical strength; they are therefore candidates as an alternative polyelectrolyte for PEFCs. ${ }^{12}$

The copolymer ratios $(m: n)$ of the partially sulfonated poly(arylene ethersulfone) (SPES) and partially sulfonated poly(arylene thioethersulfone) (SPTES) synthesized according to reported methods ${ }^{13-15}$ were estimated from the ${ }^{1} \mathrm{H}-\mathrm{NMR}$ spectra to be 80:20 for both polymers. ${ }^{13-15}$ The MWNTs were dispersed in $N, N$-dimethylacetamide (DMAc) solutions containing the polymers by sonication, as described in the Experimental section. Of interest, SPTES produced a gray solution (Figure 2a, right), whereas SPES produced a poor dispersion of the MWNTs (Figure 2a, left). Because both polymers possess similar molecular weights (viscosity of SPES and SPTES are 0.28 and $0.23 \mathrm{dl} / \mathrm{g}$, respectively, in DMAc at $30^{\circ} \mathrm{C}$ ) and a similar degree of sulfonation, we assumed that the hydrophobic nature of the thioether linkage compared with that of the ether bond reported in literature ${ }^{17}$ plays an important role during the dispersion. The importance of the hydrophobic regions during the solubilization of CNTs by the amphiphilic polymers is well recognized because the hydrophobic part is responsible for the adsorption onto the CNT surface, whereas the hydrophilic part allows for solvation to obtain wrapping of the

a
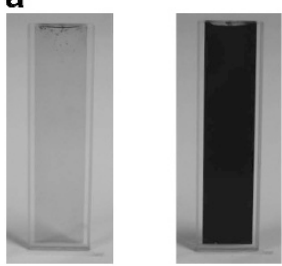

b

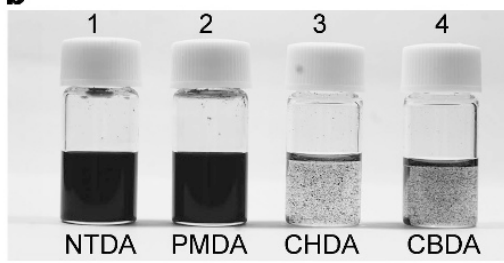

Figure 2 Photographs of DMAc supernatant solutions of the MWNTs dispersed using (a) sulfonated polyether sulfones (left: SPES and right: SPTES) and (b) SPIS (1: NTDA-BDSA, 2: PMDA-BDSA, 3: CHDA-BDSA, and 4: CBDA-BDSA).
CNT. ${ }^{18}$ In the amphiphilic SPTES and SPES, in which the sulfonic acid group serves as a hydrophilic region, the more hydrophobic nature of SPTES was preferable to form the polymer-wrapping structure because of the clear contrast in the amphiphilicity, leading to the better dispersion of CNTs.

We examined the MWNT dispersion ability of a series of SPIs synthesized through the polycondensation of BDSA with four different dianhydrides-1,4,5,8-naphthalenetetracarboxylic dianhydride (NTDA), pyromellitic dianhydride (PMDA), 1,2,4,5cyclohexyltetracarboxylic dianhydride (CHDA), and 1,2,3,4-cyclobutanetetracarboxylic dianhydride (CBDA) - which are listed in Figure 1b. As shown in Figure 2b, SPIs with an aromatic dianhydride unit-NTDA-BDSA and PMDA-BDSA — exhibited a good MWNT dispersion ability, whereas the SPIs that had an aliphatic unitCHDA-BDSA and CBDA-BDSA—showed very poor MWNT dispersion in DMAc. The $\pi-\pi$ interaction between SPIs and the CNTs is expected to play an important role in CNT dispersion using SPIs. For this study, we chose NTDA-BDSA because this polymer is one of the polyelectrolytes being extensively studied among the various SPIs as a material for PEFCs owing to its excellent chemical stability and mechanical toughness. ${ }^{19}$

The obtained supernatants from the dispersions (MWNTs in the SPTES and NTDA-BDSA solutions) were filtered and washed with DMAc to remove any unbound polymers to provide polyelectrolytewrapped MWNTs (MWNT/SPTES and MWNT/NTDA-BDSA) as gray powders after drying. The TGA of the composites measured under flowing air at a rate of $5{ }^{\circ} \mathrm{C} / \mathrm{min}$ exhibited a two-step weight reduction starting at $\sim 200^{\circ} \mathrm{C}$ and $600{ }^{\circ} \mathrm{C}$ that was attributed to the cleavage of the sulfonyl group ${ }^{20}$ and decomposition of remaining composites, respectively (Figure $3 \mathrm{a}$ ). The X-ray photoelectron spectra (XPS) of MWNT/SPTES and MWNT/NTDA-BDSA exhibited peaks that were attributed to $S_{2 p}$ and $N_{1 s}$ at $\sim 165$ and $400 \mathrm{eV}$, respectively (Figure $3 \mathrm{~b}$ ), which suggests a wrapping of SPTES and NTDA-BDSA onto the MWNTs.

\section{Anchoring of Pt on MWNTs wrapped by the polyelectrolytes} Pt-anchoring onto the composites was performed at $80^{\circ} \mathrm{C}$ using hydrogen hexachloroplatinate hexahydrate $\left(\mathrm{H}_{2} \mathrm{PtCl}_{6} \cdot 6 \mathrm{H}_{2} \mathrm{O}\right)$ and ethylene glycol as the Pt precursor and reducing agent, respectively. ${ }^{4}$ Figures $4 \mathrm{a}$ and $\mathrm{b}$ show TEM images of the composites after Pt-loading (4a; MWNT/SPTES/Pt and 4b; MWNT/BDSA-NTDA/Pt). Homogenous anchoring of the Pt-nanoparticles with a narrow diameter distribution on MWNT/SPTES/Pt $(2.0 \pm 0.47 \mathrm{~nm})$ and MWNT/ NTDA-BDSA/Pt $(2.58 \pm 0.42 \mathrm{~nm})$ was achieved (also see Supplementary Figure S1). The XPS of MWNT/SPTES/Pt exhibited peaks that were attributed to the Pt $4 \mathrm{f}$ and $\mathrm{S} 2 \mathrm{p}$ at $\sim 75$ and $165 \mathrm{eV}$,
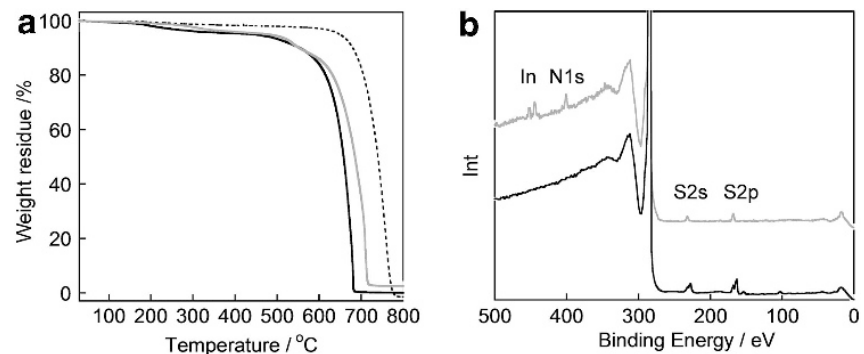

Figure 3 (a) TGA curves of the MWNT (dotted line), MWNT/STPES (black bold line) and MWNT/NTDA-BDSA (gray line). (b) XPS survey scans of MWNT/STPES (black bold line) and MWNT/NTDA-BDSA (gray line). 

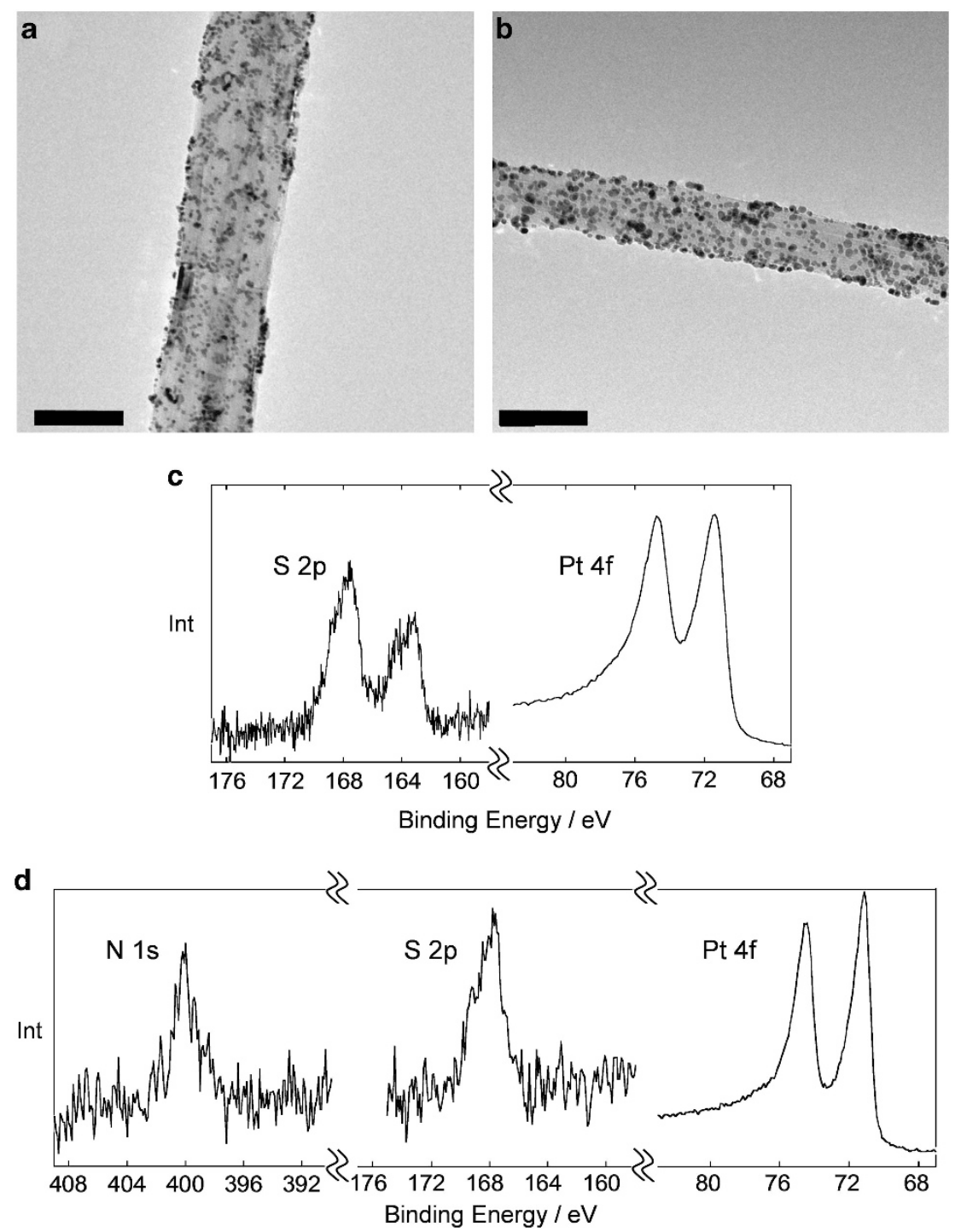

Figure 4 (a) TEM image of MWNT/SPTES/Pt. (b) TEM image of MWNT/NTDA-BDSA/Pt. (c) XPS narrow scan of MWNT/SPTES/Pt for the S2p and Pt $4 \mathrm{f}$ regions. For survey scans, see Supplementary Figure S2. (d) XPS narrow scan of MWNT/NTDA-BDSA/Pt for the S2p, $\mathrm{N} 1 \mathrm{~s}$ and Pt $4 \mathrm{f}$ regions. For the survey scan, see Supplementary Figure S2.

respectively (Figure 4c), suggesting the successful formation of an assembly of SPTES and Pt (for a wide scan, see Supplementary Figure S2).

For MWNT/NTDA-BDSA/Pt, the additional N 1s peak at $\sim 400 \mathrm{eV}$ confirmed the presence of SPI in the composites (Figure 4c). Narrow scans for the Pt $4 \mathrm{f}$ region exhibited doublet peaks at 71.1 and $74.3 \mathrm{eV}$, which were attributed to $\mathrm{Pt}^{0}\left(4 \mathrm{f}_{7 / 2}\right)$ and $\mathrm{Pt}^{0}\left(4 \mathrm{f}_{5 / 2}\right)$, respectively, indicating that the dominant valency of $\mathrm{Pt}$ is zero for both composites. $^{21}$ The TGA of the composites measured under air flow at a rate of $5{ }^{\circ} \mathrm{C} / \mathrm{min}$ exhibited a two-step weight reduction starting at $200^{\circ} \mathrm{C}$ and $400{ }^{\circ} \mathrm{C}$ that was attributed to the cleavage of the sulfonyl group ${ }^{20}$ and the decomposition of the remaining MWNT/polymer composite, respectively (Figure 5a). The onset temperature for the oxidation of the composite decreased markedly as a result of the catalytic effect of the metal nanoparticle. The $46 \mathrm{wt} \%$ of the residual corresponds to the amount of the Pt loaded onto the MWNTs, and it was calculated that as much as ca. $98 \%$ of the fed Pt was loaded onto the surfaces of the MWNTs.

It is noteworthy that the direct attachment of Pt onto the MWNTs provided a higher amount of aggregation of the Pt-nanoparticles only because of the lack of binding sites for the metal salts on the CNT surfaces. ${ }^{4}$ In previous studies, ${ }^{4}$ we revealed that the coordination of the Pt ions to the imidazole group in PBIs is a key step for efficient and homogeneous Pt-loading on CNTs. The sulfonate moiety in the polymers was expected to provide the anchoring sites on the composites, as reported for the metal-loading on CNTs wrapped by sodium dodecyl sulfonate ${ }^{22}$ and poly(stylene sulfonate). ${ }^{23}$

Figure $5 \mathrm{~b}$ shows the CV of MWNT/SPTES/Pt (black bold line) and MWNT/NTDA-BDSA/Pt (gray line). Characteristic peaks in the negative region (from -0.2 to $0.1 \mathrm{~V} \mathrm{vs} \mathrm{Ag} / \mathrm{AgCl}$ ) that are attributable to atomic hydrogen adsorption and desorption on the Pt-nanoparticle surfaces were detected. This result reveals the electronic communication between $\mathrm{Pt}$ and MWNTs. The electrochemically active surface 

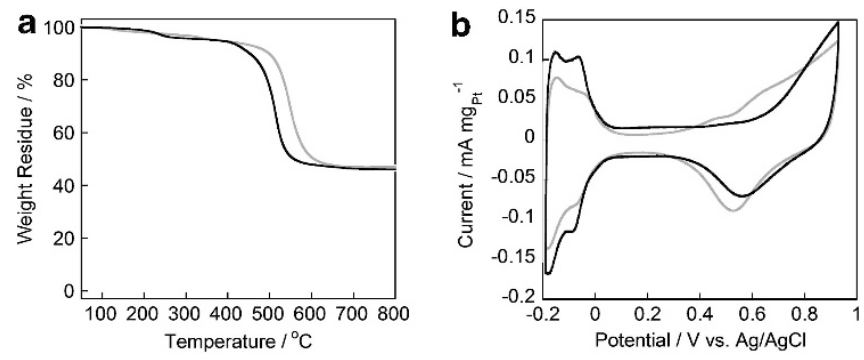

Figure 5 (a) TGA curve of MWNT/SPTES/Pt (black bold line) and MWNT/ NTDA-BDSA/Pt (gray line) measured at a rate of $5{ }^{\circ} \mathrm{C} / \mathrm{min}$ under flowing air. The loading amount of Pt on MWNT/SPTES/Pt and MWNT/NTDA-BDSA/Pt was estimated to be $46 \mathrm{wt} \%$ and $47 \mathrm{wt} \%$, respectively. (b) CVs of MWNT/SPTES/Pt (black bold line) and MWNT/NTDA-BDSA/Pt (gray line) measured in a $\mathrm{N}_{2}$-saturated $0.1 \mathrm{M} \mathrm{HClO}_{4}$ solution at a scan rate of $50 \mathrm{mV}$ per second.

area (ECSA) was calculated using Equation 1:

$$
\mathrm{ECSA}=\mathrm{Q}_{H} /(210 \times \text { Pt loading on electrode })
$$

where $\mathrm{Q}_{H}$ is the charge exchanged during the electroadsorption of $H$ on $\mathrm{Pt}$ (from -0.13 to $0.2 \mathrm{~V}$ vs $\mathrm{Ag} / \mathrm{AgCl}$ ). The amounts of $\mathrm{Pt}$ loaded onto the electrode were determined from the amounts of the composite solutions cast onto the electrodes. The ECSA of MWNT/SPTES/Pt and MWNT/NTDA-BDSA/Pt was calculated to be $42.3 \mathrm{~m}^{2} / \mathrm{g}$ for Pt and $35.6 \mathrm{~m}^{2} / \mathrm{g}$ for Pt, respectively. Such high ECSA values are the consequence of the large exposed Pt surfaces on the MWNTs and indicate the formation of an ideal triple-phase boundary structure that enables an excellent catalyst efficiency, as previously reported. ${ }^{24}$

\section{CONCLUSIONS}

We found that the sulfonated poly(arylene thioethersulfone) and SPI served as effective polymeric anchors for CNTs to immobilize Ptnanoparticles. It was revealed that the hydrophobic thioethersulfone unit functioned as a CNT anchor and that the sulfonate moiety performed as a Pt anchor for SPTES. Decreasing the hydrophobic unit allowed the delamination of the anchoring unit. However, the fully sulfonated SPI functioned as a stable Pt anchor for the MWNTs, probably as a result of the strong affinity of the fused aromatic unit in the SPI. This finding is quite useful, not only for designing the polymeric anchor but also for the development of a practical PEFC for next generation materials. Fabrication of a PEFC cell using the obtained composites as an electrocatalyst is currently in progress in our laboratory.

\section{ACKNOWLEDGEMENTS}

This work was supported by the Low-Carbon Research Network (LCnet), the Nanotechnology Network Project (Kyushu-area Nanotechnology Network), and the Global COE Program (Science for Future Molecular Systems) funded by the Ministry of Education, Culture, Sports, Science and Technology (MEXT), Japan.
1 Wu, B., Kuang, Y., Zhang, X. \& Chen, J. Noble metal nanoparticles/carbon nanotubes nanohybrids: synthesis and applications. Nano Today 6, 75-90 (2011).

2 Eder, D. Carbon nanotube-inorganic hybrids. Chem. Rev. 110, 1348-1385 (2010).

3 Peng, X., Chen, J., Misewich, J. A. \& Wong, S. S. Carbon nanotube-nanocrystal heterostructures. Chem. Soc. Rev. 38, 1076-1098 (2009).

4 Okamoto, M., Fujigaya, T. \& Nakashima, N. Design of an assembly of poly(benzimidazole), carbon nanotubes, and Pt nanoparticles for a fuel-cell electrocatalyst with an ideal interfacial nanostructure. Small 5, 735-740 (2009).

5 Hsin, Y. L., Hwang, K. C. \& Yeh, C. -T. Poly(vinylpyrrolidone)-modified graphite carbon nanofibers as promising supports for PtRu catalysts in direct methanol fuel cells. J. Am. Chem. Soc. 129, 9999-10010 (2007).

6 Wang, S., Wang, X. \& Jiang, S. P. PtRu nanoparticles supported on 1-aminopyrenefunctionalized multiwalled carbon nanotubes and their electrocatalytic activity for methanol oxidation. Langmuir 24, 10505-10512 (2008).

7 Han, X., Li, Y. \& Deng, Z. DNA-wrapped single-walled carbon nanotubes as rigid templates for assembling linear gold nanoparticle arrays. Adv. Mater 19, 1518-1522 (2007).

8 Selvaraj, V., Alagar, M. \& Kumar, K. S. Synthesis and characterization of metal nanoparticles-decorated PPY-CNT composite and their electrocatalytic oxidation of formic acid and formaldehyde for fuel cell applications. Appl. Catalysis, B: Environ. $75,129-138$ (2007)

9 Fujigaya, T., Okamoto, M. \& Nakashima, N. Design of an assembly of pyridinecontaining polybenzimidazole, carbon nanotubes and Pt nanoparticles for a fuel cell electrocatalyst with a high electrochemically active surface area. Carbon 47, 3227-3232 (2009)

10 Matsumoto, K., Fujigaya, T., Sasaki, K. \& Nakashima, N. Bottom-up design of carbon nanotube-based electrocatalysts and their application in high temperature operating polymer electrolyte fuel cells. J. Mater. Chem. 21, 1187-1190 (2011).

11 Matsumoto, K., Fujigaya, T., Yanagi, H. \& Nakashima, N. Very high performance alkali anion-exchange membrane fuel cells. Adv. Funct. Mater. 1089-1094 (2011).

12 Hickner, M. A., Ghassemi, H., Kim, Y. S., Einsla, B. R. \& McGrath, J. E. Alternative polymer systems for proton exchange membranes (PEMs). Chem. Rev. 104, 45874612 (2004)

13 Wiles, K. B., Wang, F. \& McGrath, J. E. Directly copolymerized poly(arylene sulfide sulfone) disulfonated copolymers for PEM-based fuel cell systems. I. Synthesis and characterization. J. Polym. Sci., Part A: Polym. Chem. 43, 2964-2976 (2005).

14 Schuster, M., Kreuer, K. -D., Andersen, H. T. \& Maier, J. Sulfonated poly(phenylene sulfone) polymers as hydrolytically and thermooxidatively stable proton conducting ionomers. Macromolecules 40, 598-607 (2007).

15 Wang, F., Hickner, M., Kim, Y. S., Zawodzinski, T. A. \& McGrath, J. E. Direc polymerization of sulfonated poly(arylene ether sulfone) random (statistical) copolymers: candidates for new proton exchange membranes. J. Membr. Sci. 197, 231-242 (2002)

16 Fang, J., Guo, X., Harada, S., Watari, T., Tanaka, K., Kita, H. \& Okamoto, K.-I. Novel sulfonated polyimides as polyelectrolytes for fuel cell application. 1. Synthesis, proton conductivity, and water stability of polyimides from $4,4^{\prime}$-diaminodiphenyl ether-2,2' disulfonic acid. Macromolecules 35, 9022-9028 (2002).

17 Lee, J. K. \& Kerres, J. Synthesis and characterization of sulfonated poly(arylene thioether)s and their blends with polybenzimidazole for proton exchange membranes. J. Membr. Sci. 294, 75-83 (2007)

18 Fujigaya, T. \& Nakashima, N. Methodology for homogeneous dispersion of singlewalled carbon nanotubes by physical modification. Polymer J. 40, 577-589 (2008).

19 Yin, Y., Yamada, O., Tanaka, K. \& Okamoto, K. -I. On the development of naphthalenebased sulfonated polyimide membranes for fuel cell applications. Polym. J. 38, 197-219 (2006).

20 Nakabayashi, K., Matsumoto, K., Higashihara, T. \& Ueda, M. Influence of adjusted hydrophilic-hydrophobic lengths in sulfonated multiblock copoly(ether sulfone) membranes for fuel cell application. J. Polym. Sci., Part A: Polym. Chem. 46, 7332-7341 (2008).

21 Tian, Z. Q., Jiang, S. P., Liang, Y. M. \& Shen, P. K. Synthesis and characterization of platinum catalysts on multiwalled carbon nanotubes by intermittent microwave irradiation for fuel cell applications. J. Phys. Chem. B 110, 5343-5350 (2006)

22 Tan, Z., Abe, H., Naito, M. \& Ohara, S. Arrangement of palladium nanoparticles templated by supramolecular self-assembly of SDS wrapped on single-walled carbon nanotubes. Chem. Commun. 46, 4363-4365 (2010).

23 Kongkanand, A., Vinodgopal, K., Kuwabata, S. \& Kamat, P. V. Highly dispersed pt catalysts on single-walled carbon nanotubes and their role in methanol oxidation. J. Phys. Chem. B 110, 16185-16188 (2006)

24 Munakata, H., Ishida, T. \& Kanamura, K. Electrophoretic deposition for nanostructura design of catalyst layers on Nafion membrane. J. Electrochem. Soc. 154, B1368B1372 (2007).

Supplementary Information accompanies the paper on Polymer Journal website (http://www.nature.com/pj) 